\title{
Progressive diaphyseal dysplasia: a rare bone disorder with alarming radiographs
}

\author{
Moses ML Li ${ }^{*}, \mathrm{KY}$ Chung, Alex WH Ng, KH Chiu
}

Hong Kong Med J 2020;26:538.e1-3

https://doi.org/10.12809/hkmj208498

In March 2017, a 57-year-old man was referred to our unit for left knee pain after a sprain, which resolved upon conservative treatment. Plain radiographs of the knees revealed diffuse sclerosis of bilateral femurs and tibias. A detailed history was sought to investigate the alarming skeletal pathology revealed on the plain radiographs. The patient had no history of bone pain, fever, weight loss, nor other constitutional symptoms. There was no history of malignancy. Physical examination revealed negative findings in the musculoskeletal, cardiac, pulmonary, renal, and neurological systems. Alkaline phosphatase was elevated (176 IU/L) but other blood parameters were normal, including white cell count, C-reactive protein, erythrocyte sedimentation rate, calcium, and phosphate. A skeletal survey revealed widespread sclerosis of tubular bone diaphysis of the lower extremities (Fig 1), upper extremities (Fig 2), and skull (Fig 3). Bone scintigraphy revealed increased osteoblastic activity corresponding to the locations of sclerosis (Fig 4). The characteristic radiographic features with the clinical and laboratory findings favoured a diagnosis of progressive diaphyseal dysplasia.

Sclerosing bone dysplasia is a heterogeneous

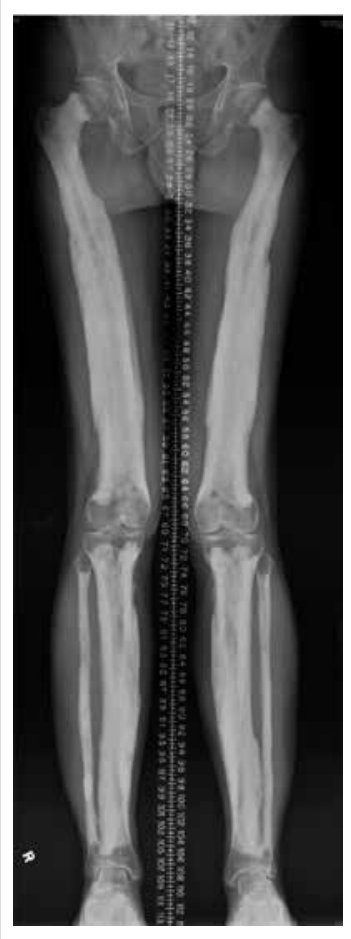

FIG I. Plain radiograph of bilateral lower limbs showing bilateral symmetrical diffuse sclerosis and cortical thickening with expansion of the diaphysis involving the femur, tibia, and fibula. Epiphyses of all the bones are spared which would be characteristic of progressive diaphyseal dysplasia

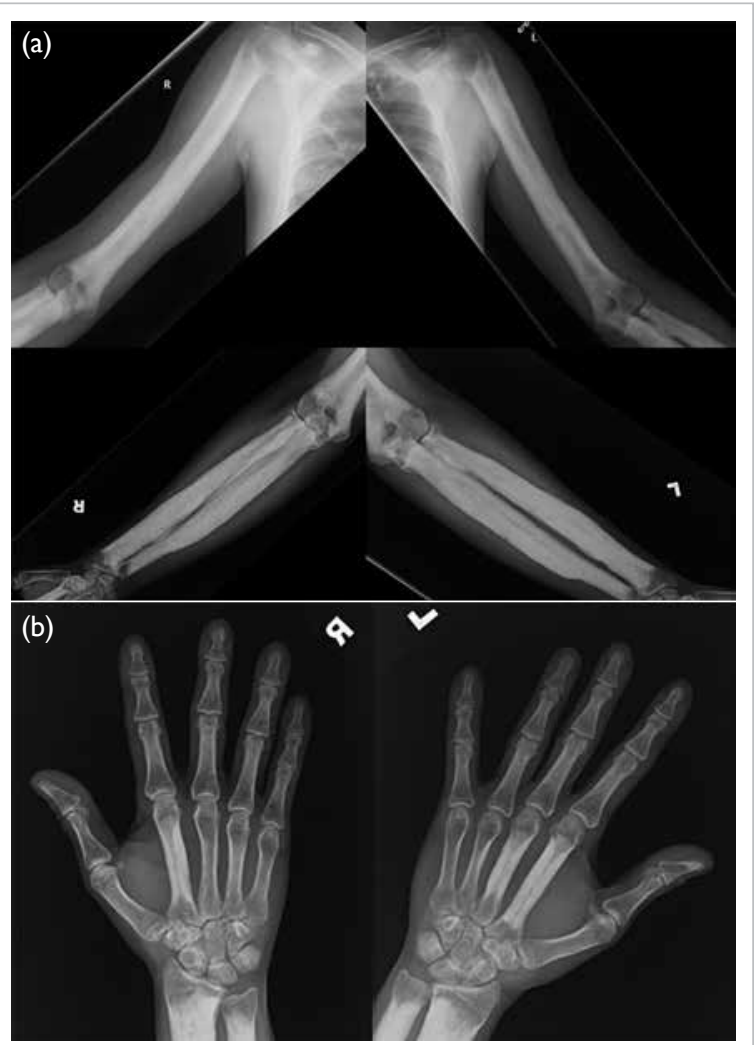

FIG 2. (a) Frontal radiographs of bilateral upper limbs showing symmetrical diffuse sclerosis and cortical thickening of the diaphysis involving the scapula, humerus, radius, and ulna. The epiphyses of these bones are also spared. (b) Frontal radiographs of the hands showing sclerosis with cortical thickening of the right second and left second to third metacarpal bones. Mild bony expansion with relative sparing of the medullary canals and epiphysis are noted

group of rare bone disorders with pathognomonic radiological features, caused by a defective ossification pathway. ${ }^{1,2}$ Progressive diaphyseal dysplasia, also known as Camurati-Engelmann disease, is a disease belonging to this entity. ${ }^{3-5}$ It is an autosomal dominant disorder due to mutation in transforming growth factor- $\beta 1$. This in turn leads to a disorder of intramembranous ossification, and results in hyperostosis. Characteristic radiological features are bilateral symmetrical fusiform sclerosis involving the diaphyses of tubular bones. The epiphyses are typically spared as these regions are formed by endochondral ossification. The lower extremities are more affected than the upper extremities. In descending order of frequency, the tibia, femur, 

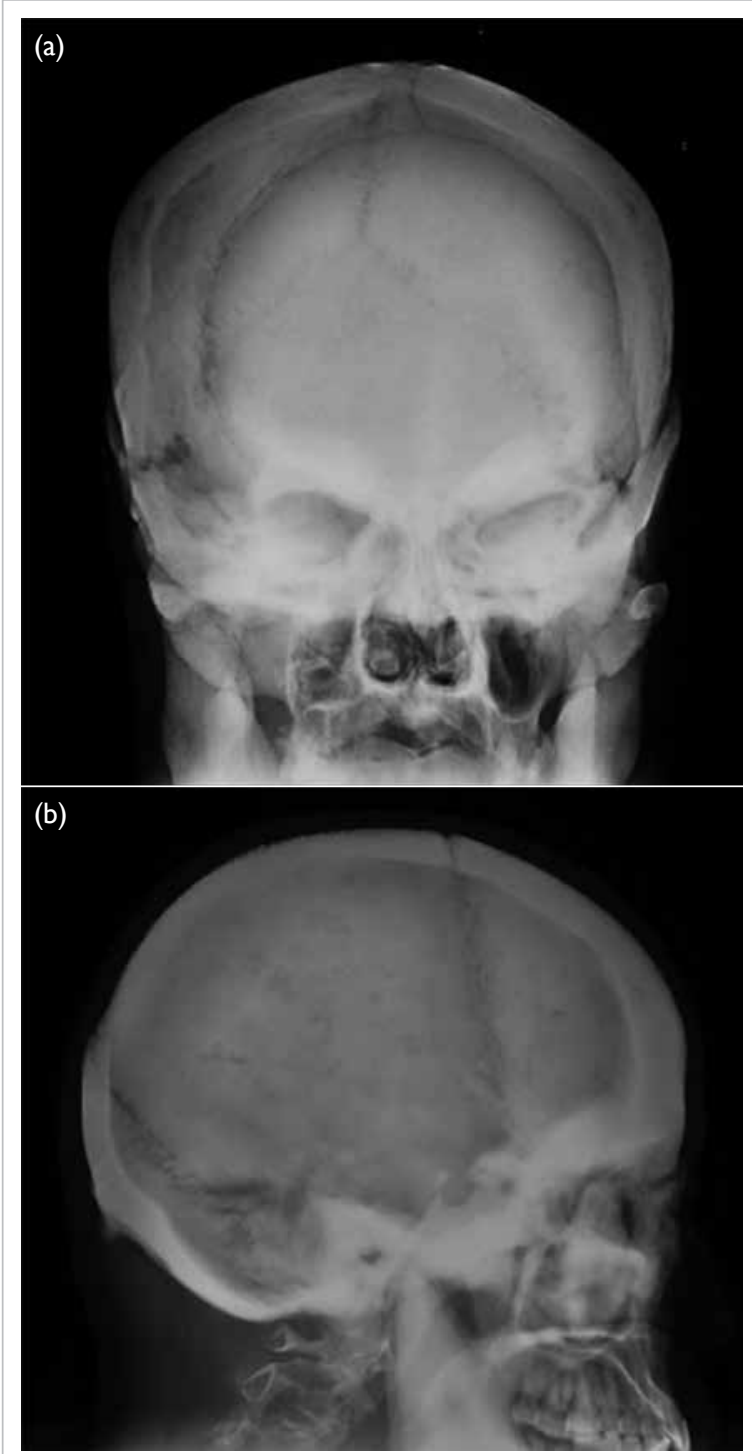

FIG 3. (a) Frontal radiograph of the skull showing sclerosis and cortical thickening of the calvaria and base of the skull. (a) Lateral radiograph of the skull showing severe sclerosis and thickening of the skull base and mandible. The cervical spine is not involved in this case

fibula, humerus, ulna, and radius are affected. ${ }^{3}$ Occasionally, the calvaria of the skull is involved. The affected bones show uneven cortical thickening of the diaphysis with hyperostosis extending in both periosteal and endosteal directions. Hyperostosis of the endosteal surface results in medullary canal narrowing.

Clinical manifestations of progressive diaphyseal dysplasia include limb pain, muscle weakness, and easy fatigability. However, our patient was completely asymptomatic. The disorder was identified incidentally during investigation of an unrelated knee sprain. Detailed systemic review revealed an absence of extraosseous manifestations

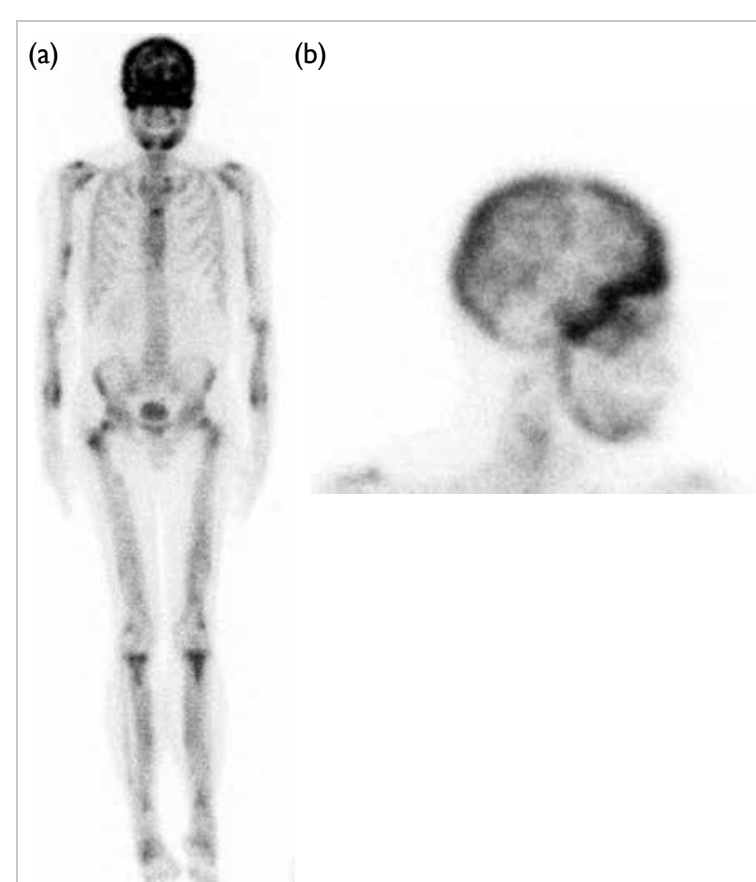

FIG 4. (a) Bone scintigraphy showing increased tracer uptake corresponding to the regions of sclerosis on plain radiographs, particularly in the skull vault and bilateral mandibles. (b) Lateral view of bone scintigraphy showing severe increased tracer uptake at the skull base

of Erdheim-Chester disease that shares similar radiological features. ${ }^{3}$ Ribbing disease (hereditary multiple diaphyseal sclerosis) usually presents with unilateral or asymmetrical involvement of the bones, and does not involve the skull vault. Osteopetrosis has epiphysis involvement and usually presents with fracture and extramedullary haematopoiesis. Although the differential diagnoses for bone sclerosis are extensive, characteristic radiographic distribution in association with clinical and laboratory findings can substantially narrow the possibilities and allow the diagnosis to be made. The age at which the diagnosis of progressive diaphyseal dysplasia is reached, the clinical manifestations of disease and the extent of radiological evidence of sclerosis are variable. ${ }^{4,5}$ Disease progression is slow and unpredictable. Treatment of the disease aims for symptomatic relief, with losartan reported to be effective in relieving limb pain based on the mechanism of down-regulation of transforming growth factor- $\beta 1$ receptor expression.

\section{Author contributions}

All authors contributed to the concept of the study, acquisition and analysis of the data, drafting of the manuscript, and critical revision of the manuscript for important intellectual content. All authors had full access to the data, contributed to the study, approved the final version for publication, and take responsibility for its accuracy and integrity. 


\section{Conflicts of interest}

All authors have disclosed no conflicts of interest.

\section{Funding/support}

This pictorial medicine paper received no specific grant from any funding agency in the public, commercial, or not-forprofit sectors.

\section{Ethics approval}

The patient was treated in accordance with the principles outlined in the Declaration of Helsinki.

${ }^{1} \mathrm{MML} \mathrm{Li}$ *, MB, ChB, MHKICBSC

${ }^{1}$ KY Chung, FRCSEd (Orth), FHKAM (Orthopaedic Surgery)

${ }^{2}$ AWH Ng, FRCR, FHKAM (Radiology)

${ }^{1}$ KH Chiu, FRCS, FHKAM (Orthopaedic Surgery)

1 Department of Orthopaedics and Traumatology, Prince of Wales Hospital, Hong Kong

${ }^{2}$ Department of Imaging and Interventional Radiology, Prince of Wales

\section{Hospital, Hong Kong}

* Corresponding author: mosesIml@gmail.com

\section{References}

1. Boulet C, Madani H, Lenchik L, et al. Sclerosing bone dysplasias: genetic, clinical and radiology update of hereditary and non-hereditary disorders. Br J Radiol 2016;89:20150349.

2. Ihde LL, Forrester DM, Gottsegen CJ, et al. Sclerosing bone dysplasias: review and differentiation from other causes of osteosclerosis. Radiographics 2011;31:1865-82.

3. Uezato S, Dias G, Inada J, Valente M, Fernandes E. Imaging aspects of Camurati-Engelmann disease. Rev Assoc Med Bras (1992) 2016;62:825-7.

4. Van Hul W, Boudin E, Vanhoenacker FM, Mortier G. Camurati-Engelmann disease. Calcif Tissue Int 2019;104:554-60.

5. Yuldashev AJ, Shin $\mathrm{CH}$, Kim YS, et al. Orthopedic manifestations of type I Camurati-Engelmann disease. Clin Orthop Surg 2017;9:109-15. 